\title{
De l'usage du pronom on dans le discours du narrateur de La Peste d'Albert Camus
}

\section{The use of the pronoun " on " in the narrator's speech of La Peste by Albert Camus}

BAUvarie Mounga [bauvarie2004@yahoo.fr]

Université de Yaoundé I, Cameroun

Patrick Dendale [patrick.dendale@uantwerpen.be]

Universiteit Antwerpen, Belgique

\section{Résumé :}

Cet article a pour but d'analyser les différents emplois du pronom on dans le discours narratif de La Peste d'Albert Camus. En effet, l'utilisation récurrente et abondante (427 occurrences) que fait le narrateur de ce pronom a attiré notre attention et nous nous attelons ainsi tout le long de notre travail à analyser et décrypter les raisons et les conséquences d'un tel phénomène. Cet article comprend quatre grands axes : les questions de recherche, la problématique et les concepts opératoires, la prise en charge et le flou narratif, l'étude de on dans le jeu polyphonique des voix narratives, les effets pragmatiques de lemploi de on.

\section{MoTS CLÉS :}

On ; narrateur ; pragmatique ; instance énonciative ; polyphonie ; narratologie

\section{Abstract:}

This article aims to analyze the different uses of the pronoun on in the narrative discourse of La Peste by Albert Camus. Indeed, the recurrent and abundant use (427 occurrences) that makes the narrator of this pronoun caught our attention and we get down and throughout our work to analyze and decipher the reasons and consequences of such a phenomenon. This article includes four main areas: research questions, issues and operational concepts, support and narrative fuzziness, studying on in the polyphonic narrative voice, the pragmatic effects of the use of $o$.

\section{KEYWORDS:}

On; narrator; pragmatics; enunciative instance; polyphony; narratology 
Etymologiquement, on a subi plusieurs transformations morphologiques avant d'avoir la forme actuelle qu'on lui connait. Alain Rey (1998 : 2458) explique à cet effet : " pron. pers. indéf., d'abord om (842) puis hom, hum (1050) et enfin on (XIIIè siècle), est issu du nominatif latin homo (dont l'accusatif hominem a donné homme) développé en position atone. A basse époque, homo est relevé dans quelques exemples comme sujet indéterminé, emploi aboutissant à sa fonction de pronom indéfini. » Essentiellement ondoyant, on est susceptible, on le sait, d'avoir des référents très différents selon les contextes. François Atlani $(1984: 23)$ va jusqu'à poser, qu' "on n'a aucune valeur référentielle ${ }^{1}$. Ces prises de position se confirment à la lecture de $L a$ Peste d'Albert Camus, où l'auteur recourt essentiellement à on pour désigner l'instance énonciative. On y constate, en effet, de façon très convaincante que les correspondances référentielles de ce pronom sont fluctuantes.

Nous voulons étudier ici comment Albert Camus, dans La Peste, exploite la perméabilité référentielle de on. ${ }^{2}$ Pour ce faire, nous nous appuierons entre autres sur l'analyse narratologique du texte littéraire ; nous essayerons de dégager les effets pragmatiques de l'emploi de on, mais avant, nous nous attarderons sur le jeu polyphonique des voix narratives générées par on.

\section{Questions de recherche, problématique et concepts opératoires}

A ce niveau, nous allons tenter de dégager toutes les questions que suscite l'emploi de on dans l'œuvre d'Albert Camus.

\subsection{Questions de recherche}

Dans La Peste, le narrateur est désigné essentiellement à travers deux pronoms : on et nous. Le pronom on compte 427 occurrences ; nous n'en compte que 65. C’est cet emploi fréquent, voire massif, que le narrateur fait du pronom indéfini on qui a attiré notre attention. A côté de cela, nous avons observé qu'avec ce pronom, on assiste tantôt à une prise en charge du discours, tantôt à une mise à distance par le narrateur dans ce discours, tantôt aux deux. Par prise en charge, nous entendons le fait que le narrateur assume son discours, dans ce sens qu'il s'en montre explicitement la source. Il indique clairement qu'il en est l'auteur. La distance du narrateur, quant à elle, renvoie au désir de celui-ci de se détacher du texte dont il est pourtant l'auteur, de

$1 \quad$ Plusieurs auteurs donnent une définition de on allant dans le même que celle proposée par François Atlani. Nous pouvons mentionner entre autres Leeman (1991 : 108) pour qui, « [...] on renvoie toujours à un ensemble de personnes, conçu non comme l'addition de personnalités distinctes, mais comme une masse indénombrable : le verbe est au singulier, et l'article indéfini qui le précède encore dans certains contextes [...] séquelle de son emploi comme nom, est un générique massif, ainsi que l'a montré Georges Kleiber.» Claire Blanche-Benveniste (2003:1) quant à elle déclare : «A examiner les emplois de On dans les usages plus courants de la langue française contemporaine, il y a lieu de sétonner de l'agilité des francophones, qui utilisent ce pronom dans des significations parfois opposés, en s'y embrouillant très rarement. " Josiane Boutet (1986 : 23) pour sa part tient à généralité cette instabilité référentielle à plusieurs pronoms. Aussi déclare-t-elle : « [...] les valeurs sémantiques ne sont pas nécessairement décidables ; l'ambiguïté est centrale dans l'interprétation des pronoms, les valeurs paradigmatiques jouent aussi un rôle dans la construction du sens. »

2 Les auteurs suivants ont fait une étude intéressante de on et nous ont aidé à mieux percevoir ce pronom : Muller (1970), Mazière (1994), Bouguerra (1999), Gjesdal (2008). 
telle sorte que le lecteur en vienne à douter à qui il faut attribuer le discours. Autrement dit, le narrateur semble ne pas assumer le discours, s'en porter garant. Prenons quelques exemples pour illustrer cet état de choses :

(1) La cité elle-même, on doit l'avouer, est laide. D’aspect tranquille, il faut quelque temps pour apercevoir ce qui la rend différente de tant d'autres villes commerçantes, sous toutes les latitudes. (LP, 11)

(2) Du moins, on ne connaît pas chez nous le désordre. Et notre population, franche, sympathique et active, a toujours provoqué chez le voyageur une estime raisonnable. $\left(\mathrm{LP}^{3}, 13\right)$

(3) A ce point du récit qui laisse Bernard Rieux derrière sa fenêtre, on permettra au narrateur de justifier l'incertitude et la surprise du docteur, puisque, avec des nuances, sa réaction fut celle de la plupart de nos concitoyens. (LP, 41)

(4) C'était en effet la mention qu'il disait faire figurer sur les feuilles d'emploi, à la suite du mot " qualification ». Lorsque vingt-deux ans auparavant, à la sortie d'une licence que, faute d’argent, il ne pouvait dépasser, il avait accepté cet emploi, on lui avait fait espérer, disait-il, une « titularisation » rapide. $(\mathrm{LP}, 47)$

Les exemples supra illustrent plusieurs façons dont le narrateur se sert de on dans son discours. Ainsi, en (1), nous avons l'impression qu'on signifie je; le narrateur semble se désigner lui-même, parler de lui. Le narrateur assume son discours. Il montre qu'il en est responsable. En (2), on s'apparenterait plutôt à un nous exclusif (je + ils). Ce que suggère fortement l'emploi de nous et notre dans la suite de la phrase. Le pronom indéfini englobe, dans cet extrait, le narrateur ainsi que ses concitoyens, les autres habitants de la ville. Pour ce qui est de l'exemple (3), on paraît y représenter le narrataire/lecteur, celui à qui le narrateur s'adresse à travers son récit. Lorsqu'on lit on permettra au narrateur de justifier l'incertitude, nous avons l'impression que on peut être remplacé par tu ou vous. Enfin, en (4), on semble avoir comme référent une personne indéfinie, quelqu'un dont l'identité n’est pas révélée ou déterminable.

Ce sont ces différents types d'emploi de on que nous voulons mettre en exergue dans cet article. Dans quel but le narrateur les utilise-t-il ? Autrement dit, quelle est l'intentionnalité qui sous-tend cette pratique ? Pourquoi le narrateur choisit-il de s'exprimer essentiellement à travers on plutôt qu'à travers d'autres pronoms ? Ou pourquoi Albert Camus n’a-t-il pas décidé tout simplement de ne pas laisser transparaître du tout les marques de l'instance énonciative? En d'autres termes, pourquoi la référence du narrateur est-elle toujours autant floue tout au long du récit? Autant de questions auxquelles nous essayerons de répondre dans cet article.

\subsection{Problématique}

On, on l'a dit plus haut, est un pronom à multiples valeurs, son référent varie en fonction du contexte dans lequel il est employé. Saunier (1996:7) le formule ainsi : 
« on " peut prendre de multiples valeurs (générique, quelconque, locuteur + allocutaire, locuteur + délocuté, locuteur, allocutaire, délocuté spécifique, délocuté indéterminé) dans la mesure où il construit une entité humaine sans prendre en compte de frontière entre les positions d'énonciateur, de co-énonciateur et de non-personne.

C'est, son sens indéfini à la base qui, lui permet de prendre diverses valeurs, de désigner différents référents en contexte. Charlotte Schapira (2006:510) estime qu’il

peut signifier une pléthore de référents qu’on ne reconnaît en discours que grâce au contexte : il peut désigner une seule personne ou plusieurs personnes à identité indéterminée, l'espèce humaine en général, un groupe de gens qui constitue la norme, l'énonciateur virtuel de l'opinion commune.

Dans cette perspective, le pronom on est ondoyant et prend la valeur que veut bien lui donner le locuteur. Nous pensons que c'est peut-être pour cette caractéristique du pronom on que le narrateur de La Peste a décidé de l'employer. Car, on lui permet, soit de prendre en charge son discours, soit de s'en distancer, d'où l'effet de polyphonie observé car il prend plusieurs valeurs. C'est en tout cas ce que nous tenterons de montrer dans notre travail. Mais avant, nous allons nous attarder sur la méthodologie employée tout au long de ce travail.

\subsection{Les concepts opératoires}

Nous convoquons essentiellement deux concepts opératoires dans cet article : la narratologie et la pragmatique. La narratologie nous semble inévitable puisqu'il est question du discours du narrateur. Guillemette et Lévesque (2006) indiquent que :

A l'aide d'une typologie rigoureuse, Genette établit une poétique narratologique, susceptible de recouvrir l'ensemble des procédés narratifs utilisés. Selon lui, tout texte laisse transparaître des traces de la narration, dont l'examen permettra d'établir de façon précise l’organisation du récit.

La narratologie nous aidera ainsi à mieux analyser les marques laissées par le pronom on et les combinaisons narratives qu'elles engendrent. Elle nous permettra de mieux cerner et appréhender le récit qu'Albert Camus met en exergue dans son œuvre.

La pragmatique, quant à elle, s'intéresse aux éléments qui permettent d'expliquer un discours en contexte. Elle ne se limite pas à la structure interne d'un énoncé, mais fait aussi appel à un certain nombre de données linguistiques ou non. Dans ce sens, Anne Reboul (1998:45) indique que « l'interprétation des énoncés est largement sous-déterminée linguistiquement. Dans cette mesure, l'interprétation d'un énoncé, si elle s'appuie sur la signification linguistique de la phrase, ne se réduit pas à celle-ci. » Une interprétation adéquate d'une performance langagière fait recours à son contexte de production et de réception. Un discours donné fait intervenir un ensemble de données de telle sorte que, comme le dit Jean Jacques Boutaud (1998 : 130), « une meilleure compréhension des processus en jeu nécessite, au-delà du linguistique la prise 
en charge du situationnel et du symbolique. » La pragmatique fait donc intervenir en plus des éléments linguistiques, des éléments extratextuels. Elle nous permettra d'étudier les effets susceptibles d'être dégagés de l'emploi contextuel et extra linguistique du pronom on dans La Peste d'Albert Camus.

Après avoir fait un point sur la problématique de on dans La Peste ainsi que la méthodologie employée pour la résoudre, il est temps de voir quelles formes il prend dans notre corpus.

\section{Prise en charge et flou narratif}

Analysons, maintenant, les différentes identités que prend l'instance énonciative de La Peste ainsi que les incidences pragmatico-stylistiques qu'elles génèrent.

\subsection{L'instance énonciative de type $1:$ le personnage générique}

L'instance énonciative s'apparente à ce niveau à un personnage qui a participé aux événements, dont l'identité est inconnue et qui est le représentant de tout un groupe de personnages. Soit les extraits de passage suivants :

(5) Rieux pensait seulement qu'il identifiait ces images à celles de son amour. Et, le jour où Rambert lui dit qu'il aimait se réveiller à quatre heures du matin et penser à sa ville, le docteur n'eut pas de peine à traduire du fond de sa propre expérience qu'il aimait imaginer alors la femme qu'il avait laissée. A quatre heures du matin, on ne fait rien en général et l'on dort, même si la nuit a été une nuit de trahison. Oui, on dort à cette heure-là, et cela est rassurant puisque le grand désir d'un cœur inquiet est de posséder interminablement lêtre qu'il aime ou de pouvoir plonger qui ne puisse prendre fin qu'au jour de la réunion. (LP, 105)

(6) Le cheval encensa une fois de plus et Rambert approuva sans passion. Le reste du déjeuner se passa à rechercher un sujet de conversation Mais tout devint très facile lorsque Rambert découvrit que le cheval était joueur de football. Lui-même avait beaucoup pratiqué le sport. On parla donc du championnat de France, de la valeur des équipes professionnelles anglaises et de la tactique en w. A la fin du déjeuner, le cheval s'était tout à fait animé et il tutoyait Rambert pour le persuader qu'il n'y avait pas de plus belle place dans une équipe que celle de demi-centre. (LP, 138-139)

Dans ces exemples, il est impossible de donner l'identité précise des locuteurs. Ce qui est clair, c'est que ces locuteurs parlent au nom de tout un groupe, d'une collectivité; ils sont parmi les personnages en présence. En (5), ce sont les habitudes des habitants de la ville d'Oran qui sont décrites. Le narrateur parle de ce qu'ils font généralement à quatre heures. En (6), le groupe décrit semble plus réduit puisqu'il s'agit de Rambert en compagnie de Raoul et Gonzalès dit le cheval, les deux hommes qui veulent l'aider à sortir clandestinement de la ville. Toutefois, il est très difficile pour nous de dire qui parle exactement entre tous ces personnages. C'est dans ce sens que Riegel, Pellat et Rioul (1994 : 197) estiment que la valeur de base de on est : 
celle d'un pronom renvoyant à une personne ou à un ensemble de personnes d'extension variable, que le locuteur ne peut ou ne veut pas identifier de façon plus précise [...]. Cette indétermination le rend apte à fonctionner comme substitut de tous les autres pour deux personnes en rejetant leur référent dans l'anonymat.

Le pronom on a l'habileté de masquer son référent. Le lecteur ne sait pas avec exactitude qui parle réellement, d’où l'adjectif générique, car le champ des référents possibles est large.

Le narrateur peut, par ailleurs, s'apparenter plus à un témoin qu'à un personnage participant aux faits relatés.

\subsection{L'instance énonciative de type 2 : le narrateur-témoin}

C'est un narrateur qui semble ne pas participer à l'histoire, mais qui intervient au cours du récit par des commentaires personnels. On a l'impression qu'il assiste juste à la scène présentée. Il a le même point de vue que la collectivité représentée dans le récit. Selon Maingueneau (1993: 111), «cette “voix" qui n'a ni la neutralité d'un narrateur anonyme ni le visage de tel ou tel personnage renvoie à la figure d'un narrateur-témoin. » C’est dire que le narrateur-témoin connaît les façons de penser du groupe de personnages décrits sans pour autant être identifiable. Examinons, à cet effet les exemples suivants :

(7) Ils étaient les malchanceux de la peste, ceux qu'elle tuait en plein espoir. Ce fut le cas du juge Othon qu'on dut évacuer du camp de quarantaine, et Tarrou dit de lui en effet qu'il n'avait pas eu de chance, sans qu'on pût savoir cependant s'il pensait à la mort ou à la vie du juge. (LP, 244)

(8) Or, vers la fin de ce mois, les autorités ecclésiastiques de notre ville décidèrent de lutter contre la peste par leurs propres moyens, en organisant une semaine de prières collectives. Ces manifestations de la piété publique devaient se terminer le dimanche par une messe solennelle placée sous l'invocation de saint Roch, le saint pestiféré. A cette occasion, on avait demandé au père Paneloux de prendre la parole. (LP, 89)

Nous constatons que dans les extraits de texte ci-dessus, la narration ne peut être attribuée à un personnage du texte, mais à la voix d'un personnage qui se présente comme faisant partie du monde évoqué. En plus, comme l'explique Maingueneau (2000:81), " grâce au « on », le narrateur apparaît bien comme à la fois à l'intérieur et à l'extérieur du monde évoqué par la narration. » De ce fait, il semble difficile de dire avec exactitude s'il est homodiégétique ou hétérodiégétique. En (7) par exemple, le narrateur raconte l'évacuation du juge Othon et on ne sait pas si il était sur les lieux ou non ; en tout cas son récit apparaît comme un témoignage. Nous ne pouvons pas dire si celui qui parle a assisté à l'évacuation du juge Othon ou s'il y a également participé. De même, à l'exemple (8), est-ce que le narrateur était présent lorsqu’on a demandé au père Paneloux de prendre la parole ? Nous ne saurions le dire avec exactitude. Aude Seron et Piotr Jan Karp (2005 : 36) déclarent que grâce à l'utilisation de on, le narrateur « [...] reste particulièrement effacé et se présente de ce fait comme un témoin objectif se contentant de re- 
later une série d'informations [...] » On a l'impression d'assister à un rapport, à un constat. En d'autres termes, le lecteur se sent comme s'il était en présence d'un procès-verbal qui relate les faits de façon objective ; c'est comme s'il y avait un journaliste ou un secrétaire qui avait pris le soin d'enregistrer les faits pour les narrés au lecteur. Le flou narratif qui se dégage du récit dans La Peste ne s'arrête pas, au contraire il semble se perpétuer avec le recours au membre quelconque d'une collectivité. Qui constitue le troisième type d'instance énonciative.

\subsection{L'instance énonciative de type 3 : le membre quelconque d'une collectivité}

Dans ce troisième cas de figure, on désigne un personnage appartenant à une communauté donnée mais dont l'identité est inconnue. Soit les extraits suivants :

(9) mais, en fait, on ne savait jamais, et Rieux préféra se taire malgré lui, il prêtait l'oreille aux rumeurs mystérieuses de la peste. On approchait du quartier de Grand et comme il était un peu surélevé, une légère brise les rafraîchissait qui nettoyait en même temps la ville de tous ses bruits. (LP, 98)

(10) Parmi les faubourgs, entre les rues plates et les maisons à terrasses, l'animation décrut et, ce quartier où les gens vivaient toujours sur leur seuil, toutes les portes étaient fermées et les persiennes closes, sans qu'on pût savoir si c'était de la peste ou du soleil qu'on entendait ainsi se protéger. (LP, 106)

Les exemples supra nous présentent un narrateur qui participe aux événements décrits sans pour autant se distinguer de la masse à laquelle il appartient. Ce n'est qu'un personnage quelconque parmi les autres. Que ce soit en (9) ou en (10), l'instance énonciative semble se présenter comme une personne faisant corps avec le groupe décrit. Elle s'apparente à l'un des membres du groupe qui a décidé de prendre la parole et de nous faire vivre les faits et événements racontés.

Nous avons décrit ci-dessus trois types d'instance énonciative que l'on retrouve dans la narration de La Peste : le personnage générique, le narrateur-témoin et le membre quelconque d'une collectivité. Ils ont tous en commun le fait qu'ils sont inidentifiables; ils racontent le récit sans que le lecteur puisse attribuer un nom connu à chacun d'entre eux. C'est du reste cette pléthore de voix dans la narration qui crée une polyphonie dans le récit.

\section{On et le jeu polyphonique des voix narratives}

A cause de la multiplicité des référents du pronom on et des voix narratives qu'il engendre, on peut dire qu'on assiste à un jeu polyphonique tout au long de l'œuvre. Nous avons l'impression que le pronom on renvoie à plusieurs voix narratives en même temps. 


\subsection{L'alternance homodiégétique/hétérodiégétique}

Evoquer le statut du narrateur, c'est s'intéresser à l'appartenance ou non de ce dernier au monde narré. A cet effet, Gérard Genette $(1972$ : 252) distingue deux types de récits : «l'un à narrateur présent comme personnage dans l'histoire qu'il raconte [...]. Je nomme le premier type, pour des raisons évidentes, hétérodiégétique et le second homodiégétique. » Le narrateur hétérodiégétique est absent comme personnage de l'histoire qu'il raconte, même s'il peut y faire des intrusions comme narrateur. Le narrateur homodiégétique, quant à lui, est présent dans l'univers diégétique même si ce n’est pas toujours sa propre histoire qu'il relate. Puisque l'identité du narrateur est souvent fluctuante, son statut alterne dans La Peste. Observons les exemples suivants :

(11) Au bout de quelques jours d'ailleurs, quand il devint évident que personne ne parviendrait à sortir de notre ville, on eut l'idée de demander si le retour de ceux qui étaient partis avant l'épidémie pouvait être autorisé. [...] Au plus grave de la maladie, on ne vit qu'un cas où les sentiments humains furent plus forts que la peur d'une mort torturée. (69)

(12) A l'intérieur, le restaurant était presque désert. Rambert alla s'asseoir à la table du fond où il avait rencontré Gonzalès, la première fois. Il dit à la serveuse qu'il attendrait. Il était dixneuf heures trente. Peu à peu les hommes rentrèrent dans la salle à manger et s'installèrent. On commença à les [Rambert et les autres hommes de la salle à manger] servir et la voûte surbaissée s'emplit de bruits de couverts et de conversations sourdes. A vingt heures, Rambert attendait toujours. On donna de la lumière. (LP, 145)

(13) Les services sanitaires purent disposer d'une liste de solliciteurs et, dès qu'une vacance venait de se produire, on avisait les premiers de la liste qui, sauf si dans l'intervalle ils étaient entrés eux aussi en vacances, ne manquaient pas de se présenter. C'est ainsi que le préfet qui avait longtemps hésité à utiliser les condamnés, à temps ou à vie, pour ce genre de travail, put éviter d’en arriver à cette extrémité. (LP, 163)

Dans le premier exemple, il s'agit d'un narrateur homodiégétique. On semble y avoir la valeur d'un nous exclusif ( je + ils), c'est-à-dire qu'il englobe le narrateur et les autres membres de la communauté. Cela revient à dire que le narrateur appartient à l'univers diégétique même s'il est difficile de donner son identité. On le voit notamment avec l'usage de l'adjectif possessif notre. Le narrateur semble donc faire partir de la diégèse. Dans les deux autres exemples, le narrateur paraît être plutôt hétérodiégétique dans la mesure où aucun élément dans les extraits de texte n'indique sa présence. Nous avons l'impression qu'il ne participe pas aux faits qu'il met en exergue. Les événements semblent se raconter d'eux-mêmes. Nous notons ainsi l'emploi des verbes tels que dit, attendait, commença, donna, purent disposer... qui donnent un aspect purement descriptif au passage. Il n'y a pas de verbes de sentiment ou de pensée. Cette alternance de statut narratif contribue à créer un brouillage narratif dans l'œuvre d'Albert Camus. 


\subsection{Brouillage narratif}

Par brouillage narratif, nous entendons ce changement continu de statut, d'identité du pronom on, qui caractérise La Peste. Le lecteur finit par ne plus savoir quel est le statut exact de ce pronom. On passe d'un personnage anonyme, à l'opinion commune puisque parfois les événements ont valeur de vérité générale. Le pronom on a comme référents je, vous, ils, nous, tout le monde. Or, c'est à l'aide ce pronom que les événements sont contés au lecteur. Il devient alors difficile de bien cerner qui est en charge du discours narratif. Fløttum, Jonassen et Norén (2007 : 48) affirment, à cet effet, que

[.... la dénotation « littérale » (en langue) de on serait donc un ensemble indéfini d'humains dont l'extension range d'un seul membre à toute l'humanité. En discours pourtant, on peut viser un individu ou un groupe plus ou moins défini, dont aussi bien le genre que le nombre et le statut énonciatif des membres peuvent être déterminés par le co(n)texte.

Le lecteur ne connaît pas réellement à qui il a affaire dans la mesure où les identités des différentes personnes en charge du récit s'entremêlent et se confondent. On est susceptible d'avoir plusieurs types de référents : du simple individu au groupe de personnes, d'une personne de sexe masculin à une autre de sexe féminin.

Ce sont tous les phénomènes narratifs sus-étudiés qui créent les « effets pragmatiques » de l'emploi de on dans La Peste d'Albert Camus.

\section{Les effets pragmatiques de l'emploi de on}

Nous allons étudier à ce niveau comment le discours narratif de La Peste exploite la plasticité de on à ses fins.

\subsection{Un rapprochement avec les lecteurs}

Le pronom on permet au narrateur de La Peste d'établir un lien étroit avec les lecteurs. Ces derniers peuvent être interpellés, ou rendus complices des événements narrés. Soit les exemples ci-après :

(14) Les maisons des malades devaient être fermées et désinfectées, les proches soumis à une quarantaine de sécurité, les enterrements organisés par la ville dans les conditions qu'on verra. (63)

(15) Et quelques-uns d'entre eux, comme Rambert, arrivaient même à imaginer, on le voit, qu'ils agissaient encore en hommes libres, qu'ils pouvaient encore choisir. (155)

(16) Et tout aurait été pour le mieux, si l'épidémie ne s'était pas étendue, comme on l'a déjà vu. (161) 
(17) Rieux savait ce que pensait à cette minute le vieil homme qui pleurait, et il le pensait comme lui, que ce monde sans amour était comme un monde mort et qu'il vient toujours une heure où on se lasse des prisons, du travail et du courage pour réclamer le visage d'un être et le cœur émerveillé de la tendresse. (236)

En (14), on peut être remplacé par nous. Le narrateur interpelle le lecteur et établit une familiarité avec lui, car il l'associe directement dans son discours. Dans les deux exemples suivants (15 et 16), le narrateur amène le lecteur à participer à la narration en relançant ainsi son discours (on le voit, on l'a déjà vu). On dirait qu'il interpelle le lecteur pour voir si celui-ci suit le rythme de la narration. Cette incise semble donc un moyen pour le narrateur de maintenir un contact, un lien avec les lecteurs. Gérard Genette (1972:262) indique à cet effet :

A l'orientation vers le narrataire, au souci d'établir ou de maintenir avec lui un contact, voire un dialogue $[. .$.$] répond une fonction qui correspond à la fois à la fonction « phatique » (vérifier le$ contact) et la fonction « conative » (agir sur le destinataire) de Jakobson.

A travers le pronom on, le narrateur introduit le lecteur dans l'univers diégétique, il fait de lui un compagnon de route, un complice. C'est comme si, tout d'un coup, il devenait autant responsable que le narrateur des faits relatés.

En (17) le pronom on peut être remplacé par n'importe quel pronom personnel, groupe nominal ou pronom indéfini. Dans ce cas, il permet au narrateur de passer d'une expérience singulière à la généralité. Autrement dit, on a comme référent je ou n’importe qui d'autre : il vient toujours une heure où on se lasse des prisons, du travail et du courage pour réclamer le visage d'un être et le cœur émerveillé de la tendresse. Dans cette phrase, ce n'est pas une expérience particulière qui est décrite, mais une expérience qui peut être faite par n'importe quel être humain, bref une observation universelle, commune à tous. Le lecteur est fortement interpellé. Alain Rabatel (2001 : 31) explique que dans cette situation,

« on » est le sujet d'authentiques perceptions représentées, en l'absence de tout focalisateurpersonnage saillant : dans cette situation, « on » est un indéfini coréférant au narrateur anonyme comme au lecteur, invité à partager la position d'un observateur anonyme.

Ainsi, on représente l'opinion commune, une doxa, un savoir partagé par chaque être humain et le lecteur peut se reconnaître dans les déclarations du narrateur. C'est comme le formule Jean-Claude Anscombre (2005: 80) :

Lorsqu'un locuteur utilise une phrase stéréotypée, il la présente comme le point de vue d’une communauté linguistique à laquelle il dit ou pas appartenir. Une telle phrase n'est donc pas à proprement parler énoncée, mais plutôt mise en place, convoquée, un peu à la façon dont les proverbes le sont pour appuyer un raisonnement.

C’est dire que quand on est employé dans une phrase stéréotypée telle que : il vient toujours une heure où on se lasse des prisons, du travail et du courage pour réclamer le visage d'un être 
et le cœur émerveillé de la tendresse, celle-ci peut être considérée comme une vérité générale, une opinion partagée par un groupe d'individus, une vision commune à un groupe de personnes données ${ }^{4}$.

\subsection{Le narrateur, témoin des événements}

Nous avons remarqué qu'avec l'emploi de on le narrateur peut se poser comme observateur des événements qu’il présente aux lecteurs. Examinons les exemples suivants :

(18) Cependant, et c'est le plus important, si douloureuses que fussent ces angoisses, si lourd à porter que fût ce cœur pourtant vide, on peut bien dire que ces exilés, dans la première période de la peste, furent des privilégiés. (LP, 75)

(19) Pendant que nos concitoyens essayaient de s'arranger avec ce soudain exil, la peste mettait des gardes et détournait les navires qui faisaient route vers Oran. Depuis la fermeture, pas un véhicule n'était entré dans la ville. A partir de ce jour-là, on eut l'impression que les automobiles se mettaient à tourner en rond. (LP, 76)

Le narrateur, à travers les exemples supra, montre qu'il participe aux faits et événements qu'il met en exergue. Il permet aux lecteurs de prendre connaissance de ce qui se passe. Il participe à l'action, les faits racontés passent par son regard. Ainsi, pour Céline Poudat (2006: 122), « [...] on est un référent bien utile dans la mesure où il permet d'introduire un objet sans en mentionner la source, ce qui permet à l'auteur d'évoquer des courants et des points de vue sans les nommer. » Le lecteur s'imprègne des faits racontés avec l'aide d'un témoin dont il n'arrive pas à déterminer l'identité.

\subsection{La neutralité et l'universalisation du récit}

Albert Camus exploite bien la nature grammaticale première de on dans La Peste : à savoir d'être un pronom indéfini. Il joue sur cette indéfinition, car tout au long de l'œuvre, il est difficile de dire qui est réellement en charge du récit. Tantôt c'est un personnage dont l'identité est inconnue, tantôt c'est un individu absent de la diégèse. Nous avons l'impression que l'auteur ne veut ni personnaliser le récit, ni y enlever toute trace de narrateur. Il voudrait que tout le monde puisse se reconnaître, s'identifier à travers le pronom on. Alain Berrendonner (1981: 58) dit à ce sujet : " on que j'appelle, faute de mieux, l'opinion publique, parce que son rôle est le plus souvent de dénoter une doxa anonyme... ». Putman (1975), quant à lui, parle de " communauté linguistique » pour désigner on ; Anscombre (2005) emploie le terme de « ON-locuteur». Chaque individu peut donc s'infiltrer dans la peau du narrateur.

4 Nous pouvons aussi citer Attal (1987: 12) qui déclare : « Bouche trou syntaxique ou substitut métalinguistique, on confère à des maximes ou à des pensées générales une très grande souplesse. » 
Cependant, à la fin de l'œuvre nous apprenons que c'est le docteur Rieux qui a rédigé tout le récit, mais a décidé de cacher son identité. Voici du reste l'extrait de texte qui révèle son identité :

(20) Au milieu des cris qui redoublaient de force et de durée, qui se répercutaient longuement jusqu'au pied de la terrasse, à mesure que les gerbes multicolores s'élevaient plus nombreuses dans le ciel, le docteur Rieux décida alors de rédiger le récit qui s’achève ici, pour ne pas être de ceux qui se taisent, pour témoigner en faveur de ces pestiférés, pour laisser du moins un souvenir de l'injustice et de la violence qui leur avaient été faite, et pour dire simplement ce qu'on apprend au milieu des fléaux, qu'il y a dans les hommes plus de choses à admirer que de choses à mépriser. (LP, 279)

On comprend à travers cet extrait de texte qu'il est question de la condition humaine dans $\mathrm{La}$ Peste. Nous pensons qu'Albert Camus a tenté d'universaliser son discours en employant presque tout le temps le pronom on. Ainsi tout lecteur peut s'y reconnaître. Chaque homme, chaque personne qui parcourt le roman peut faire siennes les souffrances qu'endure la communauté décrite dans le roman.

Terminons en essayant de répondre à la question de savoir ce que représente réellement on. A qui renvoie-t-il ?

\subsection{Qui est on?}

On a plusieurs visages dans La Peste. Il semble évoquer la voix d'une communauté qui s'exprime, parle de ses habitudes et la façon dont elle vit la tragédie qui lui arrive. On dirait donc un roman-témoignage. Le roman d'Albert Camus peut aussi être perçu comme une chronique journalistique sur les faits et les hommes (la ville d'Oran à Alger), d'où l'utilisation de on. Dans ce cas, il semble que nous soyons en présence d'un on journalistique. Nous avons vu également que le pronom on correspond à plusieurs types d'instance énonciative (personnage générique, narrateur-témoin, membre quelconque d'une même communauté) en fonction des extraits de texte.

En définitif, les valeurs de on sont multiples, même si nous avons constaté que c'est Bernard Rieux qui se cache derrière tous ces emplois.

\section{Conclusions}

L'analyse narratologique de la perméabilité référentielle de on dans le discours narratif de La Peste était l'objet principal de cet article. Nous avons vu que, dans le récit, le pronom on change constamment de référent ce qui crée une polyphonie narrative, autrement dit nous sommes en présence de plusieurs voix narratives. Difficile de dire avec exactitude qui parle, car les voix semblent se superposer. L'auteur exploite cette situation pour se rapprocher du lecteur et don- 
ner une certaine neutralité et universalité à son discours. Tous ces emplois de on auraient peutêtre pour but l'implication de tout lecteur dans les faits et la tragédie décrits par Albert Camus. L'auteur donne ainsi plusieurs visages à ce pronom indéfini.

\section{Références bibliographiques}

Anscombre, J.-C. (2005). Le On-locuteur : une entité aux multiples visages. In Dialogisme et polyphonie (pp.74-85). Bruxelles : De Boeck Université.

Atlani, F. (1984). On l'illusionniste. In La Langue au ras du texte (pp.13-29). Lille : P.U.L.

Attal, P. (1987). Emplois de ON chez la Rochefoucauld. L'Information grammaticale, 32, 12-16.

Berrendonner, A. (1981). Eléments de pragmatique linguistique. Paris : Minuit.

Blanche-Benveniste, C. (2003). Le double jeu du pronom 'on'. In P. A. Hadermann, A. Van Slijcke, \& M. Berré (Eds.), La Syntaxe raisonnée. Mélanges de linguistique générale offerts à Annie Boone à loccasion de son 60è anniversaire (pp. 43-56). Louvain-la-Neuve : De Boeck Duculot.

Bouguerra, T. (1999). L'autre je(u) du on. In J. Bres, R. Delamotte-Legrand, F. Madray-Lesigne, \& P. Siblot (Eds.), L’Autre en discours. Montpellier : Publications de l'Université de Paul Valéry, Montpellier III.

Boutaud, J. J. (1998). Sémiotique et communication. Paris : L'harmattan.

Boutet, J. (1986). La référence à la personne en français parlé : le cas de 'on'. Langage et société, 38, 19-50.

Fløttum, K., Jonassen, K., \& Norén, C., (2007). ON-pronom à facettes. Louvain-la-Neuve : De Boeck-Duculot.

Camus, A. (1947). La Peste. Paris : Gallimard.

Genette, G. (1972). Figures III. Paris : Le Seuil.

Gjesdal, A. M. (2008). Etude sémantique du pronom ON dans une perspective textuelle et contextuelle. Thèse de doctorat]. Bergen : Université de Bergen. <http://bora.uib.no/bitstream/handle/1956/3098/Anje\%20 Gjesdal.pdf?sequence $=1>$

Guillemette, L., \& Lévesque, C. (2006). La narratologie. In Hébert, L. (dir.), Signo, <http://www/signosemio. con/genette/narratologie.asp >

Leeman, D. (1991). On thème. Lingvisticae Investigationes, 15, 101-113.

Maingueneau, D. (1993). Éléments de linguistique pour le texte littéraire. Paris : Dunod.

- (2000). Instances frontières et angélisme narratif. In Langue française, 1, 74-95.

Mazière, F. (1994). On dans les dictionnaires. Faits de langue, 4, 229-236.

Muller, Ch. (1970). Sur les emplois personnels de l'indéfini on. Revue linguistique romane, 34, 48-55.

Poudat, C. (2006). Etude contrastive de larticle scientifique de revue linguistique. Thèse de doctorat. Université d'Orléans.

Putnam, H. (1975). The meaning of 'meaning'. Philosophical Papers, 2, 215-271.

Rabatel, A. (2001). La valeur de " on » pronom indéfini/pronom personnel dans les perceptions représentées. L'Information grammaticale, 8, 28-32.

Saunier, E. (1996). Identité lexicale et régulation de la variété sémantique. Thèse de doctorat. Université Paris X. 
Reboul, A. (1998). Le Discours n’est pas une catégorie scientifiquement pertinente. In Pragmatique du discours. De l'interprétation de lénoncé et l'interprétation du discours (pp. 39-71). Paris : Armand Colin.

Rey, A. (1998). Dictionnaire historique de la langue française. Paris : Dictionnaires Le Robert.

Riegel, M., Pellat, J.-Ch., \& Rioul, R. (1994). Grammaire méthodique du français. Paris : P.U.F.

Schapira, Ch. (2006). On pronom indéfini. In Indéfini et prédication (pp.507-518). Paris : PUPS.

Seron, A., \& Karp, P. J. (2005). Une analyse pragmatique de la presse boursière. Recherches en communication, 23, 23-39. 